\title{
Unusual Spread of a Penicillin-Susceptible Methicillin-Resistant Staphylococcus aureus Clone in a Geographic Area of Low Incidence
}

\author{
D. S. Blanc, ${ }^{1}$ C. Petignat, ${ }^{1}$ P. Moreillon, ${ }^{1}$ J. M. Entenza, ${ }^{1}$ \\ M.-C. Eisenring, ${ }^{2}$ H. Kleiber, ${ }^{3}$ A. Wenger, ${ }^{1}$ N. Troillet, ${ }^{2}$ \\ C.-H. Blanc, ${ }^{1}$ and P. Francioli ${ }^{1}$
}

\author{
From the ${ }^{1}$ Centre Hospitalier Universitaire Vaudois, Lausanne; \\ ${ }^{2}$ Institut Central des Hôpitaux Valaisans, Sion; \\ and ${ }^{3}$ Hôpital de la Côte, Gilly, Switzerland.
}

We describe the unusual spread of a penicillin-susceptible methicillin-resistant Staphylococcus aureus (MRSA) clone in hospitals in western Switzerland, where the incidence of MRSA is usually low. During a 2-year period, this clone had been responsible for several outbreaks and had been isolated from $>156$ persons in 21 institutions. Molecular typing by pulsed-field gel electrophoresis (PFGE) demonstrated that all of these isolates belonged to the same clone. In 1 of the outbreaks, involving 30 cases, the clone was responsible for at least 17 secondary cases. In contrast, during the period of the latter outbreak, 9 other patients harboring different MRSA strains, as assessed by PFGE, were hospitalized in the same wards, but no secondary cases occurred. These observations suggest that this clone, compared with other MRSA strains, had some intrinsic factor(s) that contributed to its ability to disseminate and could thus be considered epidemic.

During the past 15 years, methicillin-resistant Staphylococcus aureus (MRSA) has become an increasing cause of hospital infections around the world. In the early 1980s, a single strain of MRSA, characterized by phage typing, caused outbreaks in several hospitals in England and Wales [1, 2]. This strain was called epidemic (EMRSA), to distinguish it from strains that did not cause outbreaks. Since then, other epidemic strains in several countries of Europe and in Australia have been described.

EMRSA strains were mainly acquired by intensive care patients and tended to be multiresistant to antibiotics. These strains have usually been defined as epidemic only because they were detected simultaneously in several hospitals. It is postulated that some intrinsic factors, such as those involved in the colonization of the host or in the ability of the strain to survive in the environment, are responsible for this "epidemic behavior" [2]. However, there are also extrinsic factors that may contribute to the ability of a microorganism to disseminate, such as the characteristics of the source or recipient patients or control measures implemented against MRSA.

We describe in this report the unusual spread of an MRSA clone in a small geographic area, the western part of Switzer-

Received 8 July 1999; revised 10 August 1999.

This work was presented in part at the 8th European Congress on Clinical Microbiology and Infectious Diseases, held in May 1997 in Lausanne, Switzerland [abstract P443].

Grant support: This work was partially supported by a grant from the Swiss Foundation for Scientific Research (grant no. 32-45820.95).

Reprints or correspondence: Dr. Dominique S. Blanc, Division Autonome de Médecine Préventive Hospitalière, Centre Hospitalier Universitaire Vaudois, 1011 Lausanne, Switzerland (Dominique.Blanc@chuv.hospvd.ch).

Clinical Infectious Diseases 1999; 29:1512-8

(C) 1999 by the Infectious Diseases Society of America. All rights reserved. 1058-4838/1999/2906-0027\$03.00 land, where a low rate of MRSA was observed during several years of surveillance and control [3-5], except in a Geneva hospital [6]. In addition, we analyzed in more detail the epidemiology of the outbreak that occurred in 1 hospital, with a particular focus on extrinsic factors.

\section{Methods}

General setting. The hospitals involved in the present study are located in the western part of Switzerland, an area of $\sim 1.5$ million inhabitants. The university hospital of Lausanne serves as the tertiary-care center for most of the region, except Geneva, which is not included in the present study. The MRSA surveillance program was part of a larger surveillance project in Switzerland.

Surveillance and control strategies. Patients with MRSA were usually identified by surveillance of microbiological laboratory data from clinical specimens. Some were also identified by surveillance cultures of specimens obtained from patients who had been roommates of those infected or colonized with MRSA. When a cluster was suspected, patients of the ward, as well as staff members, were also screened [3].

In addition, at the university hospital of Lausanne, surveillance cultures were performed at the time of admission, when patients who were known to have been positive for MRSA were readmitted and when patients were transferred from hospitals outside the area. Surveillance culture specimens included swabs of anterior nares, throat, inguinoperineal areas, infected sites, or open wounds.

Outbreak investigation at the university hospital of Lausanne. For each patient, the following data were collected: demographic data, geographic origin, dates of admission and discharge, location and transfer within the hospital, previous hospitalization, history concerning MRSA, underlying diagnosis, and risk factors for MRSA colonization/infection and dissemination (table 1).

Microbiology. Identification of S. aureus was done by standard methods. Susceptibility testing was performed by disk diffusion on Mueller-Hinton agar with $24-\mathrm{h}$ incubation at $35^{\circ} \mathrm{C}$. Interpretation 
Table 1. Univariate analysis of potential factors for dissemination of methicillinresistant Staphylococcus aureus (MRSA) in patients harboring epidemic strain (EMRSA) vs. nonepidemic strains.

\begin{tabular}{|c|c|c|c|c|}
\hline Factor & $\begin{array}{l}\text { Patients with } \\
\text { EMRSA }\end{array}$ & $\begin{array}{l}\text { Patients with } \\
\text { other strains }\end{array}$ & OR $(95 \% \mathrm{CI})$ & $P^{\mathrm{a}}$ \\
\hline \multicolumn{5}{|l|}{ On admission } \\
\hline Indwelling urinary catheter & $3 / 29(10)$ & 2/9 (22) & $0.40(0.06-2.90)$ & NS \\
\hline Wound, decubitus ulcer & $19 / 28(67)$ & 7/9 (78) & $0.60(0.10-3.51)$ & NS \\
\hline Antibiotic therapy & $8 / 25(32)$ & 2/7 (28) & $1.18(0.19-7.42)$ & NS \\
\hline \multicolumn{5}{|l|}{$\begin{array}{l}\text { During hospitalization (before } \\
\text { MRSA detected) }\end{array}$} \\
\hline Indwelling catheter ${ }^{\mathrm{b}}$ & 28/30 (93) & $8 / 9(89)$ & - & NS \\
\hline Operation & 20/29 (69) & $6 / 9(67)$ & $1.11(0.23-5.47)$ & NS \\
\hline Wound, decubitus ulcer & $27 / 30(90)$ & $6 / 9(67)$ & $4.50(0.72-28.0)$ & NS \\
\hline Antibiotic treatment & 25/30 (83) & $6 / 9(67)$ & $2.50(0.46-13.5)$ & NS \\
\hline No. of roommates & $3 \pm 1.7$ & $1.7 \pm 1.3$ & & .01 \\
\hline No. of hospital days ${ }^{\mathrm{c}}$ & $23 \pm 23$ & $4.6 \pm 11$ & & .027 \\
\hline MRSA infection & $6 / 30(20)$ & $5 / 8(62)$ & $0.15(0.03-0.81)$ & .03 \\
\hline
\end{tabular}

criteria were from the National Committee for Clinical Laboratory Standards [7]. Resistance to oxacillin was confirmed by the screen agar test [8] and, on several occasions, by amplification of the mecA gene. Chromogenic nitrocefin disks (Cefinase; BBL Becton Dickinson, Cockeysville, MD) were used to test the phenotypic expression of $\beta$-lactamase.

Molecular typing was performed with pulsed-field gel electrophoresis (PFGE). Plugs of genomic DNA were obtained, as described elsewhere $[9,10]$ and digested with SmaI according to the manufacturer's recommendations. Electrophoresis was performed with use of the CHEF DR III system (BioRad, Hercules, CA); the conditions were $6 \mathrm{~V} / \mathrm{cm}$ for $24 \mathrm{~h}$ at $12^{\circ} \mathrm{C}$, with linear pulsed times of $1-45 \mathrm{~s}$. $\lambda$ ladder (50 kb) (New England Biolabs, Beverly, MA) was used as the molecular size standard.

The banding patterns of the different gels were analyzed with the software GelCompar (Applied Maths, Ghent, Belgium). At least 1 isolate per antibiotype and per patient was typed by PFGE. Details of the antibiogram typing method and its epidemiological value have been described elsewhere $[4,11]$. The epidemic clone was defined as all isolates showing an indistinguishable PFGE pattern and all isolates showing patterns related (with 1- to 6-band differences) to that of the major strain $[12,13]$.

Population analysis profile. The phenotypic expression of $\beta$ lactam resistance in vitro was determined, as described elsewhere [14]. In brief, different dilutions of a suspension of the test strain were spread onto agar plates containing 2-fold serial dilutions of methicillin, amoxicillin, or the combination of amoxicillin and the penicillinase inhibitor clavulanate. The population-analysis-profile curves were generated by plotting the number of colonies growing on the plates against the concentrations of the antibiotic in the plates. The well-described strain P8 [14], which is penicillinaseproducing and expresses heterogeneous resistance to methicillin, was used as a control.

Experimental endocarditis in rats. The pathogenic potential of the test strain was investigated in the rat model of endocarditis, as described elsewhere [14]. In brief, catheter-induced aortic vegeta- tions were produced in rats. Groups of rats were inoculated with saline containing different inocula $\left(10^{2}-10^{6} \mathrm{cfu}\right)$ of the test strain. These titration experiments allowed determination of the minimum inoculum of this organism required to infect 50\% $\left(\mathrm{ID}_{50}\right)$ and $90 \%$ $\left(\mathrm{ID}_{90}\right)$ of the rats. The animals were killed $24 \mathrm{~h}$ after inoculation, and quantitative cultures of the aortic vegetations were performed.

\section{Results}

Outbreaks in hospitals of western Switzerland. From January 1996 to December 1997, an increasing number of patients colonized/infected with a unique clone of MRSA was noted in western Switzerland. The relatedness between MRSA isolates was investigated by means of PFGE typing. Of 250 MRSA isolates ( 1 per patient) analyzed, 142 were indistinguishable and 14 had a related pattern (figure 1). This clone was responsible for several outbreaks in different hospitals and had been isolated from at least 136 patients and 20 staff members at 21 hospitals or nursing homes (figure 2).

This epidemic clone had been previously isolated only once, during an outbreak in May 1995 involving 1 institution, where 8 of the 14 related isolates were recovered. This suggests that the epidemic may have started in this area. Additional criteria supporting the clonal origin of these isolates were heterogenous resistance to methicillin and phenotypic susceptibility to most other antibiotics, including penicillin.

The clone was compared with other epidemic MRSA strains from Europe and other countries (Belgium clones 1, 2, and 3 [15]; Poland clones A4, A9, and B16 [16, 17]; Iberian clone I::E::A [18]; Hungarian clone III::B::A [19]; German clones [20]; United Kingdom clones EMRSA 15 and 16 [21]; and Canadian clone OE MRSA [22]). It was found to be indistinguishable from the Belgium epidemic clone 2, which was responsible in 


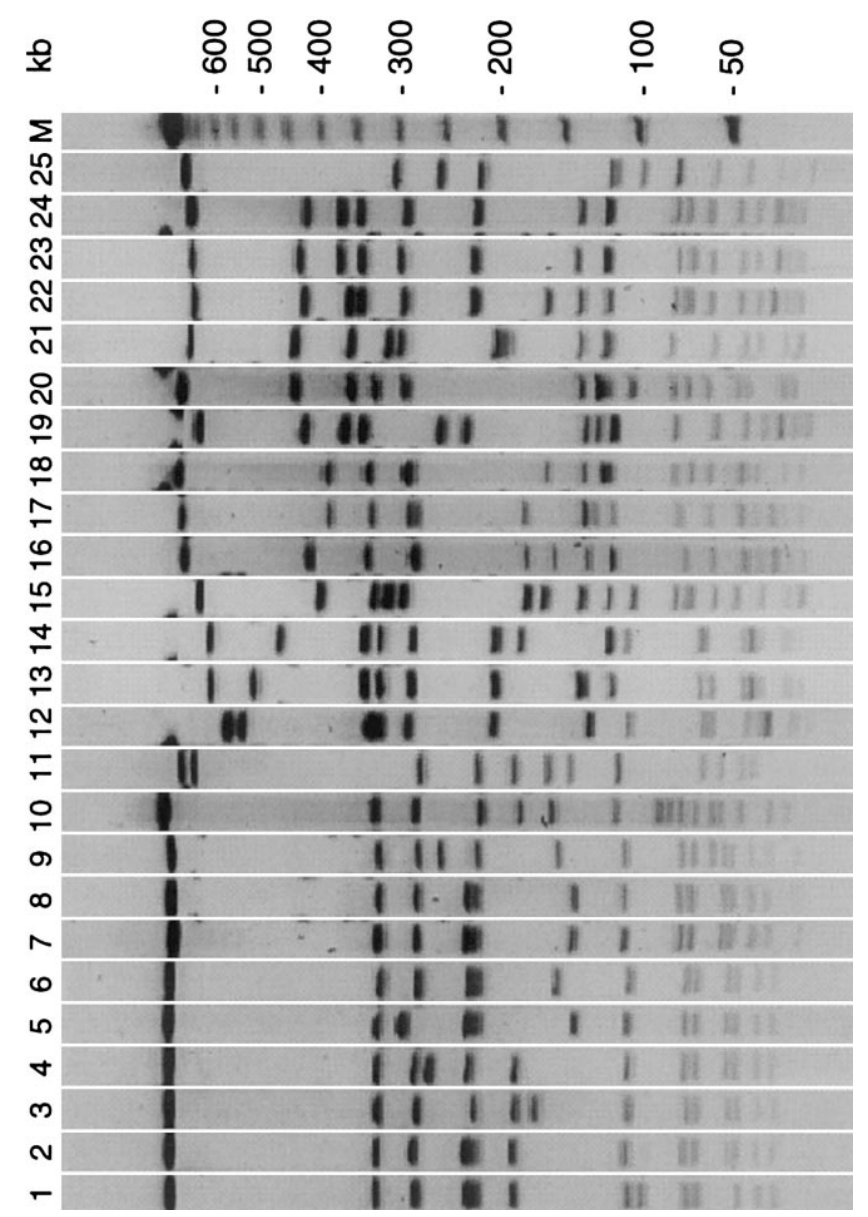

Figure 1. Pulsed-field gel electrophoresis restriction patterns of SmaI-digested DNA of western Switzerland epidemic clone of methicillin-resistant Staphylococcus aureus (MRSA) (lane 7, major pattern; lanes 1-6, examples of related patterns) and of European and Canadian epidemic clones (lane 8, Belgium clone 2; lane 9, Canadian clone OE; lane 10, Berlin clone; lane 11, UK EMRSA [epidemic methicillin-resistant $S$. aureus] 15; lane 12, south Germany clone; lane 13, Poland clone A9; lane 14, Poland clone A4; lane 15, Hungary clone III::B::A; lane 16, Hanover (Germany) clone; lane 17, southeast Germany clone; lane 18, Hanover clone; lane 19, Belgium clone 3; lane 20, Vienna clone; lane 21, Poland clone B16; lane 22, Iberian clone I::E::A; lane 23 , north Germany clone; lane 24, Belgium clone 1; and lane 25 , UK EMRSA 16). M, molecular weight marker ( $\lambda$ ladder).

1995 for $15 \%$ of MRSA isolated in Belgium [23], and was related to the OE MRSA clone, which was responsible for a large epidemic in 1996 in Ontario, Canada [22] (figure 1).

During the same period of surveillance, January 1996 to December 1997, 50 other MRSA strains were recovered from 94 patients (data not shown). Thirty-eight strains were unique, whereas 12 were recovered from $>1$ patient (2-12 patients each). With regard to transmission within hospitals, 10 clusters of only 2-7 patients were recovered. These results suggest that the MRSA strains not related to the epidemic clone had limited spreading within western Switzerland and within hospitals.
Outbreak at the university hospital of Lausanne. The outbreak started in February 1996 in the septic surgery, traumatology and rheumatology wards and involved 30 patients and 8 staff members (figure 3). During the same period, genetically different MRSA strains were isolated from 9 patients hospitalized in the same wards. All of these other strains were detected either in clinical specimens or by screening on admission.

On 4 occasions all patients of the wards were screened for MRSA, and 6 of 145 screenings were positive for MRSA; all isolates were of the epidemic clone. Staff members were also screened on 5 occasions, and of the 230 screenings, 9 were positive; 8 isolates were of the epidemic clone. Analysis of epidemiological data showed that at least 17 of 30 patients harboring the epidemic clone were secondary cases (nosocomial cases; figure 3). No secondary cases were observed with the other strains, except for 1 case involving a staff member who probably acquired the strain from a patient.

Extrinsic factors that might have contributed to the spread of MRSA were compared between the patients harboring the epidemic clone and patients harboring genetically different strains. The same infection control practices were applied for all patients during the whole period [3]: contact isolation during the period of carriage/infection, decontamination protocol for 7 days (including daily body-washing with chlorhexidine soap, intranasal mupirocin twice a day, and oral antibiotics [cotrimoxazole and rifampin]). Isolation was discontinued when surveillance cultures were negative on 2 occasions, 2 days apart.

A comparative analysis of risk factors for acquisition of epidemic versus nonepidemic strains of MRSA during hospitalization was not possible, since no secondary case occurred in the group of patients with nonepidemic strains. Factors that may play a role in MRSA dissemination were compared between patients harboring the epidemic clone and patients harboring the other strains (table 1). The results show that there were more infected versus colonized patients in the group with nonepidemic strains (5 of 8 vs. 6 of 30). They also show that the number of roommates and the duration of hospitalization before detection of MRSA were significantly greater for patients harboring the epidemic clone than for patients harboring the other strains.

Additional characteristics of the epidemic clone. Use of the disk-diffusion method showed that isolates of the clone were susceptible to penicillin, augmentin, cephalothin, cefepime, clindamycin, co-trimoxazole, erythromycin, fusidic acid, gentamicin, rifampin, and vancomycin; and were resistant to ciprofloxacin and ceftriaxone. The presence of the mecA gene was confirmed by PCR on several isolates.

The absence of $\beta$-lactamase was suggested by the chromogenic nitrocefin disk test, performed on several isolates. This was confirmed by the population analysis profile of 1 isolate (figure 4). The epidemic isolate and the strain P8, used as a control, expressed heterogeneous resistance to methicillin and contained subpopulations of bacteria able to grow on plates 


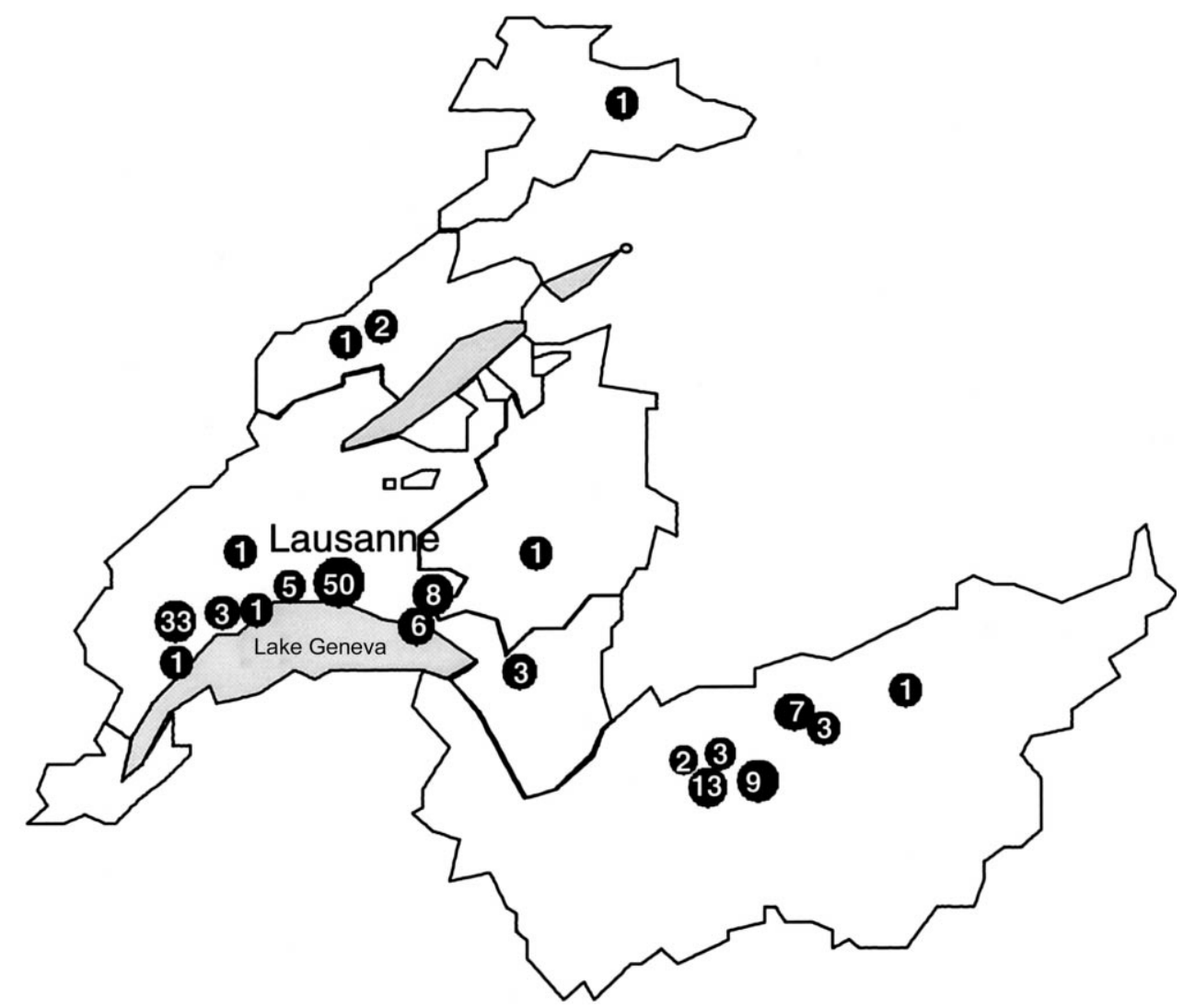

Figure 2. Geographic localization of the 21 hospitals or nursing homes (-) in western Switzerland from which epidemic clone of methicillinresistant Staphylococcus aureus was isolated during period January 1996 to December 1997 (no. in circles indicates no. of persons with epidemic clone in each particular institution).

containing methicillin ( $>125 \mathrm{mg} / \mathrm{L})$. Both strains also expressed a phenotype susceptible to amoxicillin in the presence of clavulanate. However, when plated on amoxicillin alone, the P8 strain was homogeneously resistant to the drug because of its degradation by penicillinase, whereas the susceptibility of the epidemic isolate was not altered. These results indicate that the epidemic isolate did not produce penicillinase.

The pathogenicity of 1 isolate of the epidemic clone was evaluated in the experimental model of endocarditis in rats. The minimum inocula infecting $50 \%$ and $90 \%$ of animals were $10^{3} \mathrm{cfu}$ and $10^{4} \mathrm{cfu}$, respectively. These results were similar to those for several nonepidemic strains [14], a finding suggesting that the epidemic isolate was not more pathogenic than other S. aureus strains.

\section{Discussion}

Epidemic MRSA strains were first described by investigators in England [1, 2, 24-26]. Since then, investigators in many other countries have reported the occurrence of the predominant spread of 1 strain in a defined geographic area (Germany [27,
28], Poland [16], Turkey [29], Portugal and Spain [18, 30, 31], Holland [32], and Australia [33]).

Most of the work describing epidemic strains of MRSA has been done in England, where investigators' “...experiences support the contention that some MRSA are truly epidemic, whilst others do not behave in this manner..." [2]. However, in general, a detailed analysis of extrinsic factors that might have influenced spread of the strains has not been conducted. A simplified approach was used in a Dutch study [32] and showed that, in the same setting, other MRSA strains did not spread to others patients, in contrast with the epidemic strain. However, in that study, the different patients were not hospitalized at the same time.

Many results from the present study suggest that extrinsic factors did not play a major role in the spreading of the epidemic MRSA clone. First, the clone spread rapidly in western Switzerland, a situation that had not been observed with other MRSA strains in this geographic area, where the incidence is low and where no recent changes had occurred in the health care delivery system. Second, during the investigated outbreak, the populations of patients prone to acquire MRSA from patients colonized/infected with the epidemic clone versus from 
Pts Origin Detection
pidemic MRSA clon

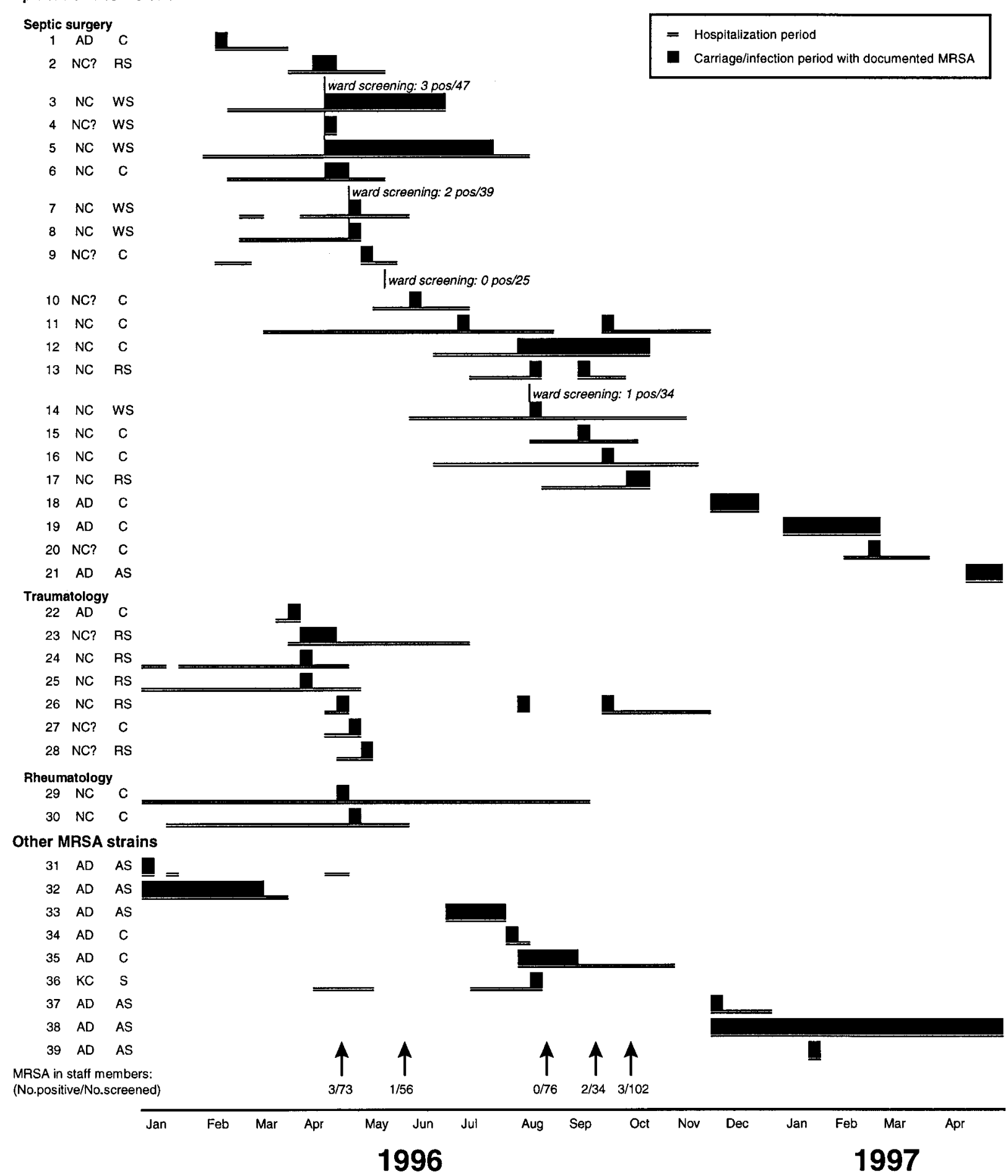

Figure 3. Outbreak of methicillin-resistant Staphylococcus aureus (MRSA) infection in septic surgery, traumatology, and rheumatology wards of university hospital in Lausanne. Column 1 indicates patient's no. Columns 2 and 3 indicate origin or time of detection of MRSA. AD, admission; AS, admission screening; C, clinical specimens; NC, nosocomial (MRSA detected during hospitalization for patients who had no record of previous carriage of or infection with MRSA and had multiple negative clinical or screening samples before MRSA detection); NC?, same as NC, but no microbiology specimen was obtained before detection of MRSA; RS, roommate screening; WS, ward screening; pos, positive. 

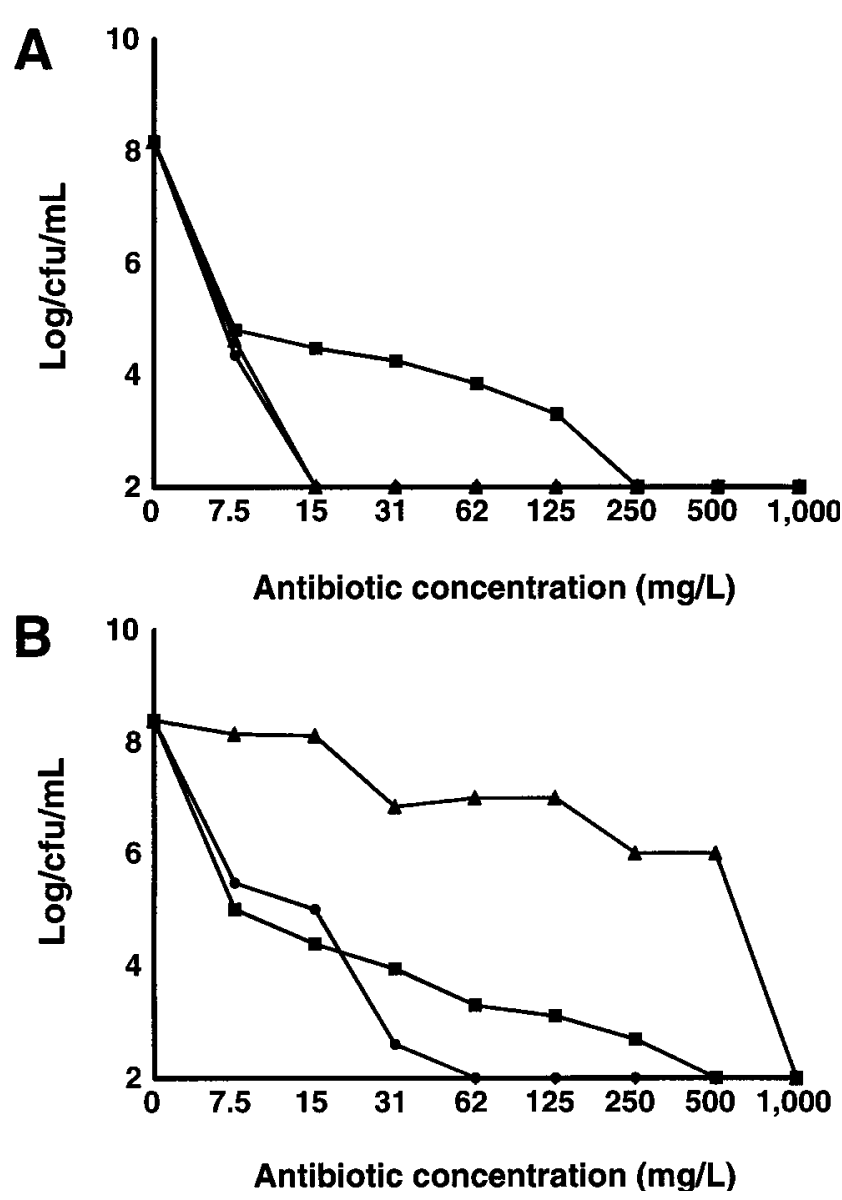

Figure 4. Population analysis profile of 1 isolate of epidemic clone (A) of methicillin-resistant Staphylococcus aureus and of control strain P8 (B). Various sizes of bacterial inocula were spread on agar plates containing increasing concentrations of methicillin $(\boldsymbol{\square})$, amoxicillin $(\boldsymbol{\Delta})$, or amoxicillin/clavulanate $(\mathbf{O})$.

patients with other strains were similar, but no secondary case occurred from the group of patients with nonepidemic strains.

Third, the control measures were identical for all patients colonized or infected with MRSA during the period of the outbreak. Fourth, the clone was recognized as epidemic in 2 other countries (Belgium [15] and Canada [22]). However, when detailed epidemiological data of the outbreak at our hospital were analyzed, it was found that the period of hospitalization before MRSA detection and the number of roommates were greater for patients with the epidemic clone than for patients with the other strains. This could explain why there were more secondary cases in the former group.

However, the duration of hospitalization before the detection of MRSA was an overestimation of the duration of unrecognized carriage, since the date of nosocomial acquisition was not known. The shorter periods of contact in the nonepidemic group could also be due to the fact that, in this group, there were more patients infected with MRSA and that most of these infections were detected on admission (admission screening, figure 3); thus they were recognized at an earlier stage. Even though these 2 factors might partly account for the differences between epidemic and nonepidemic strains, however, they are unlikely to entirely account for the magnitude of the whole epidemic in the geographic area, and it is likely that some MRSA intrinsic factors also favored the spread of this particular epidemic clone.

Multiresistance to antimicrobial agents and higher pathogenicity do not appear to account for these intrinsic factors, since the clone was susceptible to most antibiotics tested, including penicillin, and was not found to be more pathogenic than other $S$. aureus strains in the rat model of experimental endocarditis.

The present study provides additional evidence that some intrinsic factors may play a role in the epidemic behavior of some MRSA strains. Since early recognition of an epidemic strain may trigger more vigorous preventive measures that are yet to be precisely defined, the need is urgent for an easy and quick means to recognize epidemic MRSA. Further studies should be done in this field, which could involve either the detection of a genetic element responsible for the epidemicity or the setup of an international database containing the patterns of all recognized epidemic clones, with use of a standardized molecular typing system.

\section{Acknowledgments}

We are grateful to Thi Tuyet Van Le-Dao and Dorothée Raffalli for technical assistance and to all community hospitals that provided MRSA strains and epidemiological data. We thank Tony Berendt, Ian Bowler, Herminia de Lencaste, Waleria Hryniewics, Ilda Sanches, Marc Struelens, Krzysztof Trzinski, Barbara Willey, and Wolfgang Witte for providing European and Canadian epidemic strains of MRSA.

\section{References}

1. Marples RR, Cooke EM. Current problems with methicillin-resistant Staphylococcus aureus. J Hosp Infect 1988; 11:381-92.

2. Cookson BD, Phillips I. Epidemic methicillin-resistant Staphylococcus aureus. J Antimicrob Chemother 1988;21(Suppl C):57-65.

3. Lugeon C, Blanc DS, Wenger W, Francioli P. Molecular epidemiology of methicillin-resistant Staphylococcus aureus at a low incidence hospital over a 4-year period. Infect Control Hosp Epidemiol 1995; 16:260-7.

4. Blanc DS, Lugeon C, Wenger A, Siegrist HH, Francioli P. Quantitative antibiogram typing using inhibition zone diameters compared with ribotyping for epidemiological typing of methicillin-resistant Staphylo coccus aureus. J Clin Microbiol 1994;32:2505-9.

5. Francioli P, Furrer H, Pittet $\mathrm{D}$, et al. Staphylocoques dorés résistants à la méthicilline: situation et enjeux. Swiss-Noso 1995;4:25-9.

6. Pittet D, Safran E, Harbarth S, et al. Automatic alerts for methicillin-resistant Staphylococcus aureus surveillance and control: role of a hospital information system. Infect Control Hosp Epidemiol 1996; 17:496-502.

7. National Committee for Clinical Laboratory Standards. Performance stan- 
dards for antimicrobial disk susceptibility testing: approved standard M2A4. Villanova, PA: National Committee for Clinical Laboratory Standards, 1997.

8. Isenberg HD, ed. Essential procedures for clinical microbiology. Washington, DC: American Society for Microbiology, 1998:232-4.

9. Brosch R, Buchrieser C, Rocourt J. Subtyping of Listeria monocytogenes serovar $4 \mathrm{~b}$ by use of low-frequency-cleavage restriction endonucleases and pulsed-field gel electrophoresis. Res Microbiol 1991; 142:667-75.

10. Matushek MG, Bonten MJM, Hayden MK. Rapid preparation of bacterial DNA for pulsed-field gel electrophoresis. J Clin Microbiol 1996;34: 2598-600.

11. Blanc DS, Petignat C, Moreillon P, Wenger A, Bille J, Francioli P. Quantitative antibiogram as a typing method for the prospective epidemiological surveillance and control of MRSA: comparison with molecular typing. Infect Control Hosp Epidemiol 1996; 17:654-9.

12. Struelens MJ, members of the European Study Group on Epidemiological Markers. Consensus guidelines for appropriate use and evaluation of microbial epidemiologic typing systems. Clin Microbiol Infect 1996;2:2-11.

13. Tenover FC, Arbeit RD, Goering RV, et al. Interpreting chromosomal DNA restriction patterns produced by pulsed-field gel electrophoresis: criteria for bacterial strain typing. J Clin Microbiol 1995; 33:2233-9.

14. Que Y, Entenza J, Francioli P, Moreillon P. The impact of penicillinase on cefamandole treatment and prophylaxis of experimental endocarditis due to methicillin-resistant Staphylococcus aureus. J Infect Dis 1998; 177 : 146-54.

15. De Ryck R, Deplano A, Nonhoff C, Jans B, Suetens C, Struelens MJ. Molecular epidemiology and antimicrobial susceptibility of methicillinresistant Staphylococcus aureus (MRSA) in Belgium hospitals: 1992-97 [abstract P123]. Clin Microbiol Infect 1999; 5:117-8.

16. Trzcinski K, Hryniewicz W, Claus H, Witte W. Characterization of two different clusters of clonally related methicillin-resistant Staphylococcus aureus strains by conventional and molecular typing. J Hosp Infect 1994; 28:113-26.

17. Trzcinski K, Van Leeuwen W, Van Belkum A, et al. Two clones of methicillinresistant Staphylococcus aureus in Poland. Clin Microbiol Infect 1997; 3: 198-207.

18. De Lencastre H, Couto I, Santos I, Melo-Cristino J, Torres-Pereira A, Tomasz A. Methicillin-resistant Staphylococcus aureus disease in a Portuguese hospital: characterization of clonal types by a combination of DNA typing methods. Eur J Clin Microbiol Infect Dis 1994; 13:64-73.

19. De Lencastre H, Severina EP, Milch H, Konkoly Thege M, Tomasz A. Wide geographic distribution of a unique methicillin-resistant Staphylococcus aureus clone in Hungarian hospitals. Clin Microbiol Infect 1997;3: 289-96.

20. Witte W, Kresken M, Braulke C, Cuny C. Increasing incidence and wide- spread dissemination of methicillin-resistant Staphylococcus aureus in hospitals in central Europe, with special reference to German hospitals. Clin Microbiol Infect 1997; 3:414-22.

21. Lessing MPA, Jordens JZ, Bowler ICJ. When should healthcare workers be screened for methicillin-resistant Staphylococcus aureus? J Hosp Infect 1996; 34:205-10.

22. Preston M, Borczyk A, Jamieson F. Epidemic methicillin-resistant Staphylococcus aureus strain-Ontario. Canada Commun Dis Rep 1998; $24: 47-9$.

23. Aires de Sousa M, Santos Sanches I, Ferro ML, et al. International spread of multidrug-resistant methicillin-resistant Staphylococcus aureus clone. J Clin Microbiol 1998; 36:2590-6.

24. Kerr S, Kerr GE, Mackintosh CA, Marples RR. A survey of methicillinresistant Staphylococcus aureus affecting patients in England and Wales. J Hosp Infect 1990; 16:35-48.

25. Richardson JF, Reith S. Characterization of a strain of methicillin-resistant Staphylococcus aureus (EMRSA-15) by conventional and molecular methods. J Hosp Infect 1993; 25:45-52.

26. Cox RA, Conquest C, Mallaghan C, Marples RR. A major outbreak of methicillin-resistant Staphylococcus aureus caused by a new phage-type (EMRSA-16). J Hosp Infect 1995; 29:87-106.

27. Witte W, Cuny C, Braulke C, Heuck D. Clonal dissemination of two MRSA strains in Germany. Epidemiol Infect 1994; 113:67-73.

28. Schneider C, Weindel M, Brade V. Frequency, clonal heterogeneity and antibiotic resistance of methicillin-resistant Staphylococcus aureus (MRSA) isolated in 1992-1994. Zentralbl Bakteriol 1996;283:529-42.

29. Van Belkum A, Van Leeuwen W, Verkooyen R, Saçilik SC, Cokmus C, Verbrugh H. Dissemination of a single clone of methicillin-resistant Staphylococcus aureus among Turkish hospitals. J Clin Microbiol 1997; 35:978-81.

30. Aparicio P, Richardson J, Martin S, Vindel A, Marples RR, Cookson BD. An epidemic methicillin-resistant strain of Staphylococcus aureus in Spain. Epidemiol Infect 1992; 108:287-98.

31. Coello R, Jiménez J, Garcia M, et al. Prospective study of infection, colonization and carriage of methicillin-resistant Staphylococcus aureus in an outbreak affecting 990 patients. Eur J Clin Microbiol Infect Dis 1994; 13 : $74-81$.

32. Vandenbroucke-Grauls CM, Frenay HM, Van Klingeren B, Savelkoul TF, Verhoef J. Control of epidemic methicillin-resistant Staphylococcus aureus in a Dutch university hospital. Eur J Clin Microbiol Infect Dis 1991; 10: $6-11$.

33. Skurray RA, Rouch DA, Lyon BR, et al. Multiresistant Staphylococcus aureus: genetics and evolution of epidemic Australian strains. J Antimicrob Chemother 1988;21(Suppl C):19-39. 